Vilmos Prokaj, Eötvös University Budapest, Department of Probability and

Statistics, Rákóczi út 5, H-1088, Hungary. e-mail: prokaj@cs.elte.hu

\title{
MONOTONE AND DISCRETE LIMITS OF CONTINUOUS FUNCTIONS
}

\begin{abstract}
In this note we prove that for a quite large class of topological spaces every upper semi-continuous function, which is a discrete limit of continuous functions, it is also a pointwise decreasing discrete limit of continuous functions. This question was motivated by a paper of Zbigniew Grande. He asked that whether it be true for the topology of right hand continuity on the real line. He gave a partial answer showing that under some additional condition imposed on the function the answer is affirmative.
\end{abstract}

Throughout the paper we will use the following notation, for any function $f: X \rightarrow \mathbb{R}$, we denote the level set $A(f<c)=\{x \in A: f(x)<c\}$, and similarly for other relations. Recall that a sequence of function $f_{n}$ converges discretely to $f$ provided that $\cup_{n}\left(\cap_{k>n} f_{k}\right)=f$, see [2]. We will use the trick due to Urysohn, which makes it possible to define a continuous function through its level sets.

Theorem 1. Let $(X, \tau)$ be topological space, with the property that every open topological subspace is normal. Also, let $f: X \rightarrow[0,1]$ upper semi-continuous, $A \subset X$ closed subset. If $\left.f\right|_{A}$ is continuous then there is a continuous function $g: X \rightarrow[0,1]$ such that $g \geq f$ and $\left.f\right|_{A} \subset g$.

Proof. First we prove the statement for function $f: X \rightarrow[0,1)$.

To do this let $\left\{r_{k}: k \in \mathbb{N}\right\}$ be an enumeration of rational numbers in $[0,1]$, such that $r_{0}=0$ and $r_{1}=1$. We define a sequence $\left(A_{k}\right)$ such that

(1) $A_{k}$ is open,

(2) $A_{k} \subset X\left(f<r_{k}\right)$,

(3) $A_{k} \cap A=A\left(f<r_{k}\right)$,

Key Words: upper-semi continuous function, discrete limit

Mathematical Reviews subject classification: 26A03, 26A15, 26A21, 26A99

Received by the editors March 1, 1999 
(4) if $l<k$ and $r_{l}<r_{k}$ then $\overline{A_{l}} \subset A_{k}$,

(5) if $l<k$ and $r_{l}>r_{k}$ then $\overline{A_{k}} \subset A_{l}$,

(6) $\overline{A_{k} \cap A} \subset \overline{A_{k}} \cap A \subset A\left(f \leq r_{k}\right)$,

(7) and $\overline{A_{k}} \backslash A \subset X\left(f<r_{k}\right)$.

Assume that we have already defined $\left(A_{k}\right)$ for all $k$, and let

$$
g(p)=\inf \left\{r_{k}: p \in A_{k}\right\} .
$$

Property (2) ensures that $f \leq g$. From property (1), (4) and (5) $g$ is continuous and finally from property (3) $\left.f\right|_{A}=\left.g\right|_{A}$.

Now let $A_{0}=\emptyset$ and $A_{1}=X$. It is obvious that for $k=0,1$ all the above properties are fulfilled.

Assume that $A_{l}$ is defined for $l<k$ satisfying (1)-(7). Then $\tilde{X}=X \backslash A(f=$ $\left.r_{k}\right)$ is an open subset, so $\left(\tilde{X},\left.\tau\right|_{\tilde{X}}\right)$ is normal. The closed subsets of $\tilde{X}$

$$
B_{k}=\tilde{X} \cap\left(A\left(f \leq r_{k}\right) \cup \bigcup_{l<k: r_{l}<r_{k}} \overline{A_{l}}\right)
$$

and

$$
C_{k}=\tilde{X} \cap\left(X\left(f \geq r_{k}\right) \cup \bigcup_{l<k: r_{l}>r_{k}}\left(X \backslash A_{l}\right)\right)
$$

are disjoint by $(3),(4),(5),(6)$ and $(7) . \tilde{X}$ is normal so they can be separated by disjoint open sets in $\tilde{X}$, in other words: there is an open set $G_{k} \subset \tilde{X}$ such that it is larger then $B_{k}$ and its closure (in $\tilde{X}$ ) is disjoint from $C_{k}$. Put $A_{k}=X\left(f<r_{k}\right) \cap G_{k}$.

Then property (1) and (2) are clear for $A_{k}$. Since

$$
G_{k} \supset \tilde{X} \cap A\left(f \leq r_{k}\right)=A\left(f<r_{k}\right)
$$

property (3) also holds.

$A_{k} \cap \tilde{X} \subset G_{k}$ therefore $\overline{A_{k}} \cap \tilde{X}$ is disjoint from $C_{k}$. This fact implies property (7). To see (6) observe that $\overline{A_{k}} \cap A \supset \overline{A_{k} \cap A}$ and

$$
\overline{A_{k}} \cap A \subset \overline{G_{k}} \cap A \subset\left(A \backslash C_{k}\right) \cup(A \backslash \tilde{X}) \subset A\left(f<r_{k}\right) \cup A\left(f=r_{k}\right) .
$$

We have to show that (4) and (5) also hold. Since $\overline{A_{l}} \backslash A \subset X\left(f<r_{l}\right)$ for $l<k$ we have that if $r_{l}<r_{k}$ then 
$\overline{A_{l}}=\left(\overline{A_{l}} \backslash A\right) \cup\left(\overline{A_{l}} \cap A\right) \subset X\left(f<r_{l}\right) \cup A\left(f \leq r_{l}\right) \subset X\left(f \leq r_{l}\right) \subset X\left(f<r_{k}\right)$.

On the other hand,

$$
\overline{A_{l}} \subset \overline{A_{l}} \backslash A \cup A\left(f \leq r_{l}\right) \subset \tilde{X},
$$

so by the choice of $G_{k}, \overline{A_{l}} \subset G_{k}$. It follows that $\overline{A_{l}} \subset G_{k} \cap X\left(f<r_{k}\right)=A_{k}$ and (4) is shown.

Finally, to see (5) let $l<k$ such that $r_{l}>r_{k}$ and observe that

$$
\overline{A_{k}} \backslash A \subset \overline{G_{k}} \cap \tilde{X}
$$

which is disjoint from $C_{k}$ so $\overline{A_{k}} \backslash A$ does not meet $\tilde{X} \backslash A_{l}$. So

$$
\overline{A_{k}}=\left(\overline{A_{k}} \backslash A\right) \cup\left(\overline{A_{k}} \cap A\right) \subset A_{l} \cup A\left(f \leq r_{k}\right) \subset A_{l} \cup A\left(f<r_{l}\right) \subset A_{l} .
$$

So the sequence $\left(A_{n}\right)$ can be defined and the theorem is proved for the special case when $f$ does not take the value 1 .

For the general case let $f^{\prime}=(1 / 2) f$ and $g^{\prime}$ be a continuous function such that $\left.f^{\prime}\right|_{A} \subset g^{\prime}$ and $f^{\prime} \leq g^{\prime}$. Then $g=\min \left(2 g^{\prime}, 1\right)$ is continuous, $g$ extends $\left.f\right|_{A}$ and $f \leq g$. This completes the proof.

Of course not all topological space satisfies the condition of the previous theorem, e.g let $X=2^{\aleph_{1}}$ be the topological power of the compact discrete space $\{0,1\}=2$, where $\aleph_{1}$ is the first uncountable cardinal. $X$ is compact and Hausdorff, so normal. However, it is also a folklore among topologists that for any point $e \in X$ the subspace $X \backslash\{e\}$ is not normal. This shows that the condition we have found in Theorem 1 is not necessary, since in the paper of Grande [1], Theorem 1 — proposed by the referee - states the same implication for $\sigma$-compact spaces.

When someone deals with limits of continuous functions, perfectly normal spaces behave nicely. The next lemma states that for perfectly normal spaces the previous theorem applies.

Lemma 2. Let $(X, \tau)$, be a perfectly normal topological space, if $G \in \tau$ then $\left(G,\left.\tau\right|_{G}\right)$ is normal.

Proof. Let $F_{1}$ and $F_{2}$ closed subsets of $X$ such that $F_{1} \cap F_{2} \cap G=\emptyset$. We have to prove that there are open sets $S_{1}, S_{2} \subset G$ such that $S_{1} \cap S_{2}=\emptyset$, $F_{i} \cap G \subset S_{i}$ for $i=1,2$.

$S_{1}$ and $S_{2}$ will be level sets of a continuous function $f: G \rightarrow[0,2]$. So first we define this function. Let $A=F_{1} \cup F_{2}$. Since the space is perfectly normal 
there are continuous functions $f_{i}: F_{i} \rightarrow[0,1]$ such that $F_{i}\left(f_{i}=1\right)=F_{i} \backslash G$. Put $f=f_{1} \cup\left(2-f_{2}\right)$. $f$ is a function on $A$ since both $f_{1}$ and $f_{2}$ takes 1 on $A \backslash G$. It is continuous because it is bounded and its graph is closed in $X \times \mathbb{R}$. $f$ can be extended to $X$ to a continuous function, we denote this extension also $f$.

Now $S_{1}=G(f<1)$ and $S_{2}=G(f>1)$ are open subsets of $G$ and separate $F_{1} \cap G$ and $F_{2} \cap G$.

We can state somewhat more.

Lemma 3. Let $(X, \tau)$ be a topological space such that for any upper semi-continuous function $f: X \rightarrow[0,1]$ and closed set $A$ there is a continuous function $g: X \rightarrow[0,1]$ such that $\left.f\right|_{A} \subset g$ and $f \leq g$, provided that $\left.f\right|_{A}$ is continuous. Then $X$ is normal.

Proof. Let $F_{1}$ and $F_{2}$ be disjoint closed sets. Put $f=\chi_{F_{1}}$ the characteristic function of $F_{1} . f$ is upper semi-continuous, $\left.f\right|_{F_{1} \cup F_{2}}$ is continuous, so by the hypothesis there is a continuous function $g$ such that $\left.f\right|_{F_{1} \cup F_{2}} \subset g$. Clearly, $g$ separates $F_{1}$ and $F_{2}$.

The two lemmas and the theorem have the following

Corollary 4. Let $(X, \tau)$ be a perfect topological space (i.e. every open set is $F_{\sigma}$ in $\left.X\right)$. Then the following three statements are equivalent

(i) for any upper semi-continuous function $f: X \rightarrow[0,1]$ and closed set $A$ there is a continuous function $g: X \rightarrow[0,1]$ such that $\left.f\right|_{A} \subset g$ and $f \leq g$, provided that $\left.f\right|_{A}$ is continuous,

(ii) $(X, \tau)$ is normal,

(iii) for any open set $G \subset X,\left(G,\left.\tau\right|_{G}\right)$ is normal.

The following statement is almost obvious from the definitions and it was also used in [1].

Lemma 5. Let $X$ be a normal topological space and $f: X \rightarrow \mathbb{R}$ be a function. $f$ is a discrete limit of continuous functions if and only if there is a sequence $\left(A_{k}\right)$ of closed subset of $X$ such that $\left.f\right|_{A_{k}}$ is continuous and $\cup_{k} A_{k}=X$

Theorem 6. Let $(X, \tau)$ be topological space, with the property that every open topological subspace is normal. Also, let $f: X \rightarrow \mathbb{R}$ be an upper semi-continuous function which is discrete limit of a sequence of continuous functions. Then there is a sequence $\left(f_{n}\right)$ of continuous functions such that $f_{n+1} \leq f_{n}$ and $f_{n}$ tends to $f$ discretely. 
Proof. By usual trick we trace back the general case to the one when actually $f: X \rightarrow(0,1)$. Composing every functions with a continuous increasing bijection of $(0,1)$ to $\mathbb{R}$ it is clear that it is enough to show that $f_{n}$ can be chosen in such a way that beside the listed properties in the statement we have $f_{n}: X \rightarrow(0,1)$.

By lemma 5 there are closed sets $A_{n}$ such that $\left.f\right|_{A_{n}}$ continuous and $\cup_{n} A_{n}=$ $X$. Forming finite unions we can also assume that $A_{n} \subset A_{n+1}$. Then by theorem 1 there are continuous functions $g_{n}^{\prime}: X \rightarrow(0,1]$ such that $\left.f\right|_{A_{n}} \subset g_{n}^{\prime}$ and $g_{n}^{\prime} \geq f$. Put

$$
g_{n}=\sum_{k=n}^{\infty} \frac{1}{2^{k+1-n}} g_{k}^{\prime}
$$

Then $g_{n}: X \rightarrow(0,1), g_{n} \geq f$ and it extends $\left.f\right|_{A_{n}}$, So $f_{n}=\min _{k \leq n} g_{k}$ is pointwise decreasing and tends to $f$ discretely.

The topology examined by Zbigniew Grande in [1] is the topology of the continuity from the right, i.e. for which the sets $[a, b), a<b$ constitutes a base. In what follows this topology will be denoted by $\tau$.

Although, the topology $\tau$ is not second countable, it is hereditary Lindelöf therefore every topological subspace is normal. Sometimes such space is called completely or hereditary normal. It is also well known that $\tau$ is perfect.

In [1] Grande defined three properties, (1), (1)' and (2) of upper from the right semicontinuous functions such that if $f$ is a discrete limit of continuous functions from the right then it satisfies (1). He proved that (1) implies (1)' which is equivalent to (2), and that (1)' does not imply (1). Eventually he proved that any upper from the right semi continuous function fulfilling property (1)' is a pointwise decreasing discrete limit of a sequence of from the right continuous functions. He asked whether condition (1)' can be replaced with condition (1). The answer follows from the above theorem. To see this, we repeat first property (1) from [1]. All the topological notions without the prefix $\tau$ refer to the natural topology of the real line.

A function $f:[0,1) \rightarrow \mathbb{R}$ satisfies property (1) if every non-empty perfect set $A$ has a non-empty portion $I \cap A$ such that $\left.f\right|_{B \cap I}$ is $\tau$-continuous, where $B$ is the set of not $\tau$-isolated points of $A$.

Lemma 7. Let $f:[0,1) \rightarrow \mathbb{R}$ be a function satisfying property (1). Then there is a sequence $A_{n}$ of $\tau$-closed sets such that $\left.f\right|_{A_{n}}$ is $\tau$-continuous and $\cup_{n} A_{n}=[0,1)$.

Proof. We will use the following simple facts.

1. for a closed set $A$ the set of the accumulation points of $A$, denoted by $A^{\prime}$ is a perfect subset of $A$ and $A \backslash A^{\prime}$ is at most countable. 
2. If $A$ is any set then the set of $\tau$-isolated points of $A$ is at most countable.

By transfinite recursion we define a sequence $\left\{I_{\alpha}: \alpha<\xi\right\}$ of open intervals (in $[0,1)$ ) with rational endpoints, beside this we will define a sequence $C_{\alpha}$ of the same type such that each $C_{\alpha}$ is at most countable.

Let $H_{0}=[0,1) . H_{0}$ is perfect in the natural topology of $[0,1)$. So by property (1) there is a non-empty open interval $I_{0}$ with rational endpoint such that $\left.f\right|_{I_{0}}$ is $\tau$-continuous.

Assume that we have already defined $I_{\beta}$ for $\beta<\alpha$. Let $S_{\alpha}=[0,1) \backslash$ $\bigcup\left\{I_{\beta}: \beta<\alpha\right\}$ and $H_{\alpha}=S_{\alpha}^{\prime}$ be the set accumulation points of $S_{\alpha}$.

$H_{\alpha} \subset S_{\alpha}$, since this later is closed, and $H_{\alpha}$ is perfect. If $H_{\alpha}$ is empty then we do not continue the sequence. Otherwise, property (1) provides an interval $I_{\alpha}$ that meets $H_{\alpha}$. Put

$$
C_{\alpha}=\left(S_{\alpha} \backslash H_{\alpha}\right) \cup\left\{x \in H_{\alpha}: x \text { is } \tau \text {-isolated }\right\} .
$$

Then $C_{\alpha}$ is countable and $\left.f\right|_{I_{\alpha} \cap\left(H_{\alpha} \backslash C_{\alpha}\right)}$ is $\tau$-continuous.

So the sequence $\left\{I_{\alpha}, C_{\alpha}: \alpha<\xi\right\}$ is defined. Observe that the map $\alpha \mapsto I_{\alpha}$ is injective, since $I_{\alpha}$ meets a set $\left(H_{\alpha}\right)$ which is disjoint from all $I_{\beta}, \beta<\alpha$. This means that $\xi$ is a countable ordinal.

Note, also, that $H_{\alpha} \backslash C_{\alpha}$ is $\tau$-closed. Therefore $I_{\alpha} \cap\left(H_{\alpha} \backslash C_{\alpha}\right)$ is $\tau$ - $F_{\sigma}$, as well as $\bigcup\left\{C_{\alpha}: \alpha<\xi\right\}$. To finish the proof let us choose $\tau$-closed sets $A_{\alpha, n}$ such that

$$
I_{\alpha} \cap\left(H_{\alpha} \backslash C_{\alpha}\right)=\bigcup_{n=1}^{\infty} A_{\alpha, n} .
$$

And finally let us enumerate the countable family

$$
\left\{A_{\alpha, n}: \alpha<\xi, n \in \mathbb{N}\right\} \cup\left\{\{p\}: p \in \cup_{\alpha} C_{\alpha}\right\}
$$

into a sequence $\left(A_{n}\right)$.

It is clear from the construction that $\cup_{n} A_{n}=[0,1)$ and $\left.f\right|_{A_{n}}$ is $\tau$-continuous for each $n$.

Corollary 8. Let $f:[0,1) \rightarrow \mathbb{R}$ be a function. $f$ is a discrete limit of $\tau$ continuous functions if and only if $f$ satisfies property (1).

Proof. It was proved in [1] that if $f$ is a discrete limit of $\tau$-continuous functions then it satisfies property (1).

To prove the opposite implication, let $A_{n}$ be a sequence of $\tau$-closed sets such that $\left.f\right|_{A_{n}} \tau$-continuous and $\cup_{n} A_{n}=[0,1)$. The topology $\tau$ is normal so there are $\tau$-continuous functions $f_{n}$ such that $\left.f\right|_{\cup_{k=1}^{n} A_{k}} \subset f_{n}$. It is clear that $f_{n}$ tends to $f$ discretely.

Now we can answer in the affirmative the problem posed by Grande. 
Corollary 9. Assume $f:[0,1) \rightarrow \mathbb{R}$ satisfies property (1) and $\tau$-upper semicontinuous. Then there is a sequence of $\tau$-continuous functions $f_{n} \geq f_{n+1}$ tending to $f$ discretely.

Proof. By lemma 7 there are $\tau$-closed sets $A_{n}$ such that $\left.f\right|_{A_{n}} \tau$-continuous and $\cup_{n} A_{n}=[0,1)$. By lemma 5 we can apply theorem 6 and the proof is complete.

\section{References}

[1] Zbigniew Grande, On A.C. Limits and Monotone Limits of Sequences of Jump Functions, Real Analysis Exchange, 25.2(2000), -. appear).

[2] Ákos Császár and Miklós Laczkovich, Discrete and Equal Baire Classes, Acta Math. Hung., 55(1990), 165-178. 
